\title{
The age-old puzzle of suicide: How does the mind get so dark that it wants total obliteration?
}

\author{
Hong Kong Nguyen
}

February 14, 2021

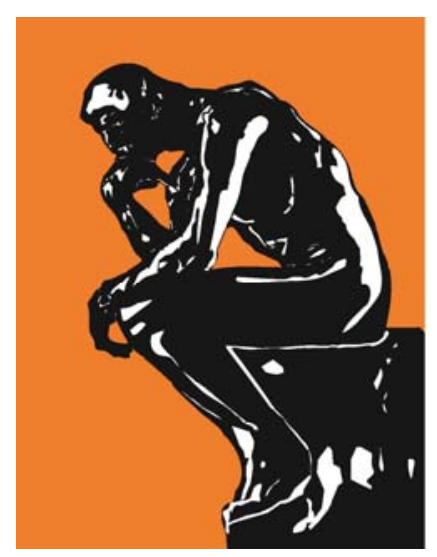

AISDL

One of the most complicated topics I have wrestled with ever since I was a Philosophy major in college is suicide. Not because I ever contemplated it, but because it strangely came up quite often in my studies. I was baffled when the tragic nature of suicide was glorified in the Iliad by Homer: Ajax, the son of Telamon, killed himself with a sword out of shame after having slaughtered the cattle of the Greeks in a bloodlust [1]. Suicide out of shame, honor, protest, or even patriotism is indeed not new - the ancient Greeks wrote epic poems about it, the Japanese samurai (and later kamikaze pilots) made it part of their code of conduct, and the extremist Islamic terrorists today blew themselves up in acts of suicide terrorism [2]. Even in ordinary cases where one may commit violence and harm against oneself, suicide may result from panic, desperation, grief, and perhaps some unfathomable pain. During my four years of undergraduate, I knew of at least two classmates who died by suicide, and the events puzzled me to this day. What kind of pain were they going through that I couldn't see or help with? Is ending one's life the answer to the difficult questions about human existentialism?

It turns out I wasn't alone in my quest to untangle the enigma behind suicidal ideation and behavior. For decades, the topic has apparently haunted social psychologists, neurologists, and mental health experts alike. The literature is populated with empirical 
studies on the neurobiology of suicide [3], the socio-economic determinants of suicide, the correlates to suicidal ideation, and the major analytical theories aimed at explaining the thought process toward suicide. Standing out among these theories are the Interpersonal Theory of Suicide (IPTS), the Integrated Motivational-Volitional Model (IMV), the Three-step Theory (3ST), and the Fluid Vulnerability Theory (FVT). The explanatory power of these models lies in their emphasis on the distinction between the suicidal ideation process and the attempted suicide process. Some key concepts of the four "ideation-to-action" models are capture as follows.

- The IPTS, first developed by Thomas Joiner [4] and further examined by [5-7], for the first time distinguishes between the act of suicide and the capability for suicide. In other words, the desire to kill oneself - determined by "perceived burdensomeness" and "social alienation"-is deemed insufficient, for without developing the ability to be, for instance, fearless of pain, injury, and death through repeated self-injuries or accidental injuries, the final act would not be realized.

- The IMV, developed by Rory O'Connor [8], takes into account the complex and multifaceted arrays of suicide-related risk factors, thus, integrating a tripartite framework of biology, psychology, and sociology. By separating background factors from trigger events through three such perspectives, the author has moved beyond the conventional psychiatric approach to suicide and managed to identify additional factors such as signals of defeat and humiliation.

- The 3ST, proposed by David Klonsky and Alexis May [9], hypothesizes that (i) a combination of pain and hopelessness leads to thoughts of suicide, (ii) a lack of social connectedness may escalate suicidal ideation among those suffering from pain and hopelessness, and (iii) the progression toward suicidal attempt is driven by a combination of dispositional, acquired, and practical contributors to the capacity for suicide.

- The FVT, a cognitive approach theorized by Rudd David [10], delves deeper into suicide risk assessment and integrates the cognitive and affective systems with motivational and behavioral systems.

Of these models, the first three focus on the factors influencing the ideation process and its progression to attempted suicide. The FVT instead explores suicidal ideation as a kind of information processing. Framing suicide in this angle is intriguing, for suicide is part of a considerable volume of information each individual processes on a daily basis; how suicide gets to be the dominant train of thought in this processing is worth inquiring. I suggest that integrating the model of the mindsponge $[11,12]$ and Bayesian inference [13-15] could supplement the existing theories on suicidal ideation- 
to-action, adding a new kind of depth to our understanding of suicidal information and thought processing.

The mindsponge mechanism expounds on an individual's information process through a multi-filtering system and layers of information domains. An individual's core mindset is at the center, deciding whether to accept or filter out emerging information, beliefs and/or values. The mechanism, inspired by the analogy of a sponge absorbing water quickly and sloshing water right back out, suggests that the human mind can process information in a similar manner. Within this framework, it becomes quite apparent that, for those who become suicidal, the thought must have arisen so strongly, so invasively that it has entered into the core mindset, which for certain reasons must have become tolerant and accepting of suicidal values and ideas rather than ejecting them outright. Following the Bayesian information update process, the suicidal ideation-toaction progression is captured as: suicide-related information is introduced to an individual and is constantly updated, filtered, analyzed, and eventually either accepted or rejected.

Through this lens, dark thoughts are seen as constantly attacking the mind, which has already become vulnerable and open toward such darkness. Darkness begets darkness. Perhaps this is how this downward spiral toward total obliteration comes about. How do we bring light into this darkness? Understanding how humans process information is the first step toward understanding those who become accepting of suicide.

\section{References}

1. Brouwers, J. (n.d.) The suicide of Ajax. Available online:

https://www.ancientworldmagazine.com/articles/suicide-ajax/ (accessed on 2018, February 14).

2. Atran, S. (2003). Genesis of Suicide Terrorism. Science, 299, 1534-1539, doi:10.1126/science.1078854.

3. Mann, J. (1998). The neurobiology of suicide. Nature Medicine, 4, 25-30, doi:10.1038/nm0198-025.

4. Joiner, T. (2005). Why people die by suicide; Harvard University Press: Cambridge, MA,

5. Van Orden, K.A.; Cukrowicz, K.C.; Witte, T.K.; Joiner Jr, T.E. (2012). Thwarted belongingness and perceived burdensomeness: construct validity and psychometric properties of the Interpersonal Needs Questionnaire. Psychological Assessment, 24, 197. 
6. Van Orden, K.A.; Witte, T.K.; Cukrowicz, K.C.; Braithwaite, S.R.; Selby, E.A.; Joiner Jr, T.E. (2010). The interpersonal theory of suicide. Psychological Review, 117,575 .

7. Van Orden, K.A.; Witte, T.K.; Gordon, K.H.; Bender, T.W.; Joiner Jr, T.E. (2008). Suicidal desire and the capability for suicide: Tests of the interpersonalpsychological theory of suicidal behavior among adults. Journal of Consulting Clinical Psychology, 76, 72.

8. O'Connor, R.C. (2011). Towards an integrated motivational-volitional model of suicidal behaviour. International handbook of suicide prevention: Research, policy and practice, 1, 181-198.

9. Klonsky, E.D.; May, A.M. (2015). The Three-Step Theory (3ST): A New Theory of Suicide Rooted in the "Ideation-to-Action" Framework. International Journal of Cognitive Therapy, 8, 114-129, doi:10.1521/ijct.2015.8.2.114.

10. Rudd, M.D. (2006). Fluid Vulnerability Theory: A Cognitive Approach to Understanding the Process of Acute and Chronic Suicide Risk. In Cognition and suicide: Theory, research, and therapy., American Psychological Association: Washington, DC, US, 2006; 10.1037/11377-016pp. 355-368.

11. Vuong, Q.H. (2016). Global mindset as the integration of emerging socio-cultural values through mindsponge processes: A transition economy perspective. In Global Mindsets, Kuada, J., Ed. Routledge; pp. 123-140.

12. Vuong, Q.H.; Napier, N.K. (2015). Acculturation and global mindsponge: an emerging market perspective. International Journal of Intercultural Relations, 49, 354-367.

13. Vuong, Q.-H.; Ho, M.-T.; La, V.-P. (2019). 'Stargazing' and p-hacking behaviours in social sciences: some insights from a developing country. European Science Editing, 45, 54-55.

14. Vuong, Q.-H.; La, V.-P.; Nguyen, M.-H.; Ho, M.-T.; Tran, T.; Ho, M.-T. (2020). Bayesian analysis for social data: A step-by-step protocol and interpretation. MethodsX, 7, 100924, doi:https://doi.org/10.1016/j.mex.2020.100924.

15. La, V.-P.; Vuong, Q.-H. (2019). bayesvl: Visually Learning the Graphical Structure of Bayesian Networks and Performing MCMC with 'Stan'. The Comprehensive R Archive Network (CRAN). Available online: https://cran.rproject.org/web/packages/bayesvl/index.html version 0.8 .5 (officially published on May 24, 2019) (accessed on February 13, 2019). 